\title{
Predictive value of common clinical and laboratory parameters in predicting response to anti thyroid drug therapy in graves hyperthyroidism a prospective study
}

\author{
Sripathy Gopalakrishnan, Pradeep Kumar Chugh, Mitrabasu, Jyoti Sethi, Neelam Chhillar, Sankar Rajan*
}

\author{
Scientist 'E', DEBEL,DRDO, Bangalore \\ Scientist ' $F$ ' and Joint Director, INMAS, DRDO, Delhi \\ Scientist ' $F$ ' and Joint Director, INMAS, DRDO, Delhi \\ Technical Officer 'A', INMAS, DRDO, Delhi \\ Associate Prof, IHBAS, Delhi \\ *Corresponding author E-mail: rsankar@gainhealth.org
}

\begin{abstract}
Purpose: Graves' hyperthyroidism (GH) is commonly treated by antithyroid drugs (ATD). This study was undertaken to identify those clinical factors that could predict sustained remission with ATD so as to avoid unwanted therapy.

Approach: Subjects ( $\mathrm{n}=134)$ diagnosed with GH were consecutively recruited. Carbimazole was administered for 18 months as per titration regimen and subsequently followed up for upto 1 month to detect relapse of thyrotoxicosis.

Results: In final analysis of 115 subjects, 61 remained in remission while thyrotoxicosis recurred in 54 subjects during follow-up. Parameters associated with remission for at least 6 months were: smaller goiter, lower free triiodothyronine (FT3) values at onset and euthyroidism in response to ATD within 3-6 months.

Conclusions: $50 \%$ of patients went into remission with carbimazole therapy for 18 months. Subjects who fail to achieve euthyroidism with 6 months of ATD should be offered radioiodine or surgery.
\end{abstract}

Keywords: Antithyroid drugs, euthyroidism, goiter, Graves'disease, and thyrotoxicosis.

\section{Introduction}

Graves' disease is a common endocrine disorder and accounts for nearly three-fourths of cases of hyperthyroidism [1]. The essential goal of management in Graves' hyperthyroidism (GH) is to reduce the hyper secretion of thyroid hormones and achieve permanent euthyroidism. There are three forms of treatment - antithyroid drugs, radioactive iodine and surgery - each of which is not exclusive of the other. None of the three forms of therapy guarantee the objective of permanent euthyroidism. The choice of first line therapy varies in different regions of the world, as reported by Wartofsky et al and Glinoer et al [2],[3]. Though radioiodine is gaining widespread acceptance as first choice for adult patients in North America, antithyroid drugs continue to be the mainstay of therapy in other parts of the world. Studies differ widely regarding the long term remission rates reported after a course of antithyroid drugs (ATD) and ranges from 30-70\% [4],[5]. In order to improve the outcome after antithyroid drug therapy, studies have attempted to determine factors which will help to identify those patients who will enter remission after ATD. Some of the clinical and laboratory parameters such as goiter size, antithyroid antibody levels, Human Leucocyte Antigen (HLA) subtypes, Thyrotropin Releasing Hormone (TRH) suppression test have been found to influence response to ATD in studies by Schleusner et al and Vitti et al, but could not accurately predict outcome to ATD therapy [6], [7] .

Almost all studies on treatment outcome or factors predicting remission in GH pertain to populations outside the Indian sub-continent who differ in ethnic origin and iodine nutrition. We undertook a study to determine the rate of remission after ATD and whether we could identify factors that will help to predict remission. The study was conducted in patients with Graves' hyperthyroidism attending the Thyroid Clinic of the Institute of Nuclear Medicine and Allied Sciences (INMAS), Delhi, a tertiary level referral centre. 


\section{Methods}

The thyroid outpatient department at INMAS is a tertiary level referral center and is attended by patients suspected to have thyroid disorders referred from all over northern India. If thyrotoxicosis is suspected clinically, a set of investigations (free triiodothyronine (FT3), free thyroxine (FT4), thyroid stimulating hormone (TSH), antimicrosomal antibodies (AMc) and radioactive iodine uptake (RAIU)) are carried out to confirm diagnosis and under lying cause. The basis of diagnosis of GH was the initial thyrotropin and thyroid hormone levels, radioactive iodine uptake at 2,6 and 24 hours and thyroid scan at 24 hours. Those diagnosed with GH for the first time were included for the study. Anyone with past history of ATD or other forms of therapy for hyperthyroidism were excluded. Patients were consecutively recruited into the study after taking an informed written consent. The study was approved by the research advisory panel of the Defence Research and Development Organization under the Ministry of Defence, Government of India.

\subsection{Protocol of ATD}

A course of ATD (carbimazole) for 18 months was offered in the hope of achieving remission and patients were advised to follow up at INMAS at regular intervals ( 8 weeks after initiation of therapy and every 8 to 12 weeks thereafter) for clinical and lab evaluation. Dose of ATD were titrated according to FT3 /FT4 levels done at each visit so as to maintain biochemical euthyroidism. Initial dose was $30 \mathrm{mg}$ of carbimazole per day given in two divided doses for those weighing above $40 \mathrm{~kg}$ and $20 \mathrm{mg}$ for those weighing less than $40 \mathrm{~kg}$. Ophthalmopathy was managed conservatively with eye protection measures. Those patients who developed hypersensitivity or could not tolerate the drugs were taken out of the study and were offered alternative options: surgery or radioactive iodine therapy. After the ATD course was completed, they were followed up further to detect relapse. Subjects who continued to be euthyroid off ATD were considered to have achieved remission from GH. For outcome evaluation in this study, those who were in remission for at least six months after stopping antithyroid drugs were grouped as responders and the remainder as non-responders.

\subsection{Follow up visits}

Clinical evaluation at each visit was done to assess for presence of symptoms and signs suggestive of thyrotoxicosis. Goiter size was graded as per the WHO/UNICEF/ICCIDD classification [8] and ophthalmopathy as per NOSPECS classification [9]. Blood samples were collected from the subjects at each visit, serum was separated and estimation of serum FT3, FT4 and TSH was carried out. Clinical examination findings and levels of serum thyroid hormones were used to guide the therapy of the subjects.

\subsection{Diagnostic criteria for $\mathbf{G H}$}

GH was defined as the presence of biochemical hyperthyroidism (either elevate d serum FT4, FT3 or both and undetectable TSH) together with 2 of the following: Diffuse Goitre, Presence of thyroid anti-microsomal antibodies (a titer of 1: 400 or higher was considered significant) Raised radioactive iodine uptake at $24 \mathrm{~h}$ of more than $30 \%$ and diffuse goiter on scan at 24 hours.

\subsection{Investigation methods}

Radioiodine uptake (RAIU) was conducted by administering $50 \mu \mathrm{Ci}$ (micro curies) of 131I in the fasting state and the $131 \mathrm{I}$ counts were measured at $2 \mathrm{hr}$ and $24 \mathrm{hr}$ and the uptake was calculated in comparison to counts taken over thigh. The scan was done after 24 hours using a linear collimator to confirm the presence of diffuse goiter [10].

FT3 and FT4 were estimated by the electrochemiluminescence (ECL) technique using commercially available kits from Roche Diagnostics (Mannheim, Germany) with Elecsys 1010 analyzer. The analytical sensitivity and total precision values for FT3, FT4 assays were $0.400 \mathrm{pM} / \mathrm{L} \& 2.2 \%$ and $0.30 \mathrm{pM} / \mathrm{L} \& 2.7 \%$ respectively. The laboratory reference ranges were: FT3 (3.7-7.2 pM/L) and FT4 (12.0-23.0pM/L). The intra-assay coefficients of variation for the assays were $4.7 \%$ and $1.4 \%$ respectively.

Serum TSH was estimated using immunoradiometric (IRMA) kits supplied by Bhabha Atomic Research Centre(BARC), Mumbai, India with the reference range being 0.25-5.0 $\mu \mathrm{IU} / \mathrm{ml}$ and intra- assay CV being $1.9 \%$. Antimicrosomal antibodies (AMc) were measured using semiquantitative hemagglutination kits (Serodia) obtained from Fujerobio Inc, Tokyo, Japan. Tests were considered positive only if titers were higher than 1:400. 


\subsection{Factors recorded at diagnosis}

Gender, age at diagnosis, eye signs, goiter size, anti- thyroid antibody titre and concentration of serum thyroid hormones and thyrotropin, radioactive iodine uptake values at 2 hours and 24 hours and scan at 24 hours.

\subsection{Defined Outcome after a course of ATD}

Successful- euthyroid for at least 6 months after withdrawal of ATD.

Failed - persistence or relapse after a full course of ATD and progression to RAI or surgery.

\subsection{Statistics}

Descriptive statistics and data analysis were carried out using the statistical software SPSS version 15.0 (SPSS Inc.Chicago, USA). Numerical data were expressed as mean \pm standard deviation (SD). Data was analyzed using Chisquare test on $2 \mathrm{X} 2$ contingency tables or by Mann-Whitney test as was appropriate. Factors contributing to the prediction of response to treatment were identified using logistic regression.

\section{Results}

One hundred and thirty four consecutive patients who were newly diagnosed with Graves' hyperthyroidism were recruited into the study. None of the subjects included into the study had received any antithyroid drugs before attending our clinic. The mean age, gender distribution and salient clinical characteristics of the subjects studied are given in table 1.

\begin{tabular}{cccc}
\multicolumn{4}{c}{ Table 1: Clinical features of study population of graves' hyperthyroidism } \\
\hline Characteristic & Total & Responders & Non Responders \\
\hline Subjects (n) & 115 & 61 & 54 \\
Age (yrs) Mean \pm SD & $34.32 \pm 10.65$ & $34.26 \pm 11.67$ & $34 \pm 9.46$ \\
Male/Female Ratio & $33 / 82(1: 2.48)$ & $15 / 46(1: 3)$ & $18 / 36(1: 2)$ \\
Goitre (grade) & $\mathrm{I}=7$ & $\mathrm{I}=5$ & $\mathrm{I}=2$ \\
& $\mathrm{II}=74$ & $\mathrm{II}=44$ & $\mathrm{II}=30$ \\
& $\mathrm{III}=34$ & $\mathrm{III}=12$ & $\mathrm{III}=22$ \\
FT3 (pmol/L) & $23.91 \pm 10.32$ & $23.26 \pm 13.89$ & $31.01 \pm 12.73$ \\
FT4 (pmol/L) & $59.22 \pm 17.6$ & $59.17 \pm 18.21$ & $66.45 \pm 15.13$ \\
\hline & Normal range for FT3: $3.7-7.2 \mathrm{pM} / \mathrm{L}$ Normal range for FT4: $12.0-23.0 \mathrm{pM} / \mathrm{L}$ &
\end{tabular}

All those recruited in to the study were started on antithyroid drug as per our study protocol. Seven subjects developed drug hypersensitivity and were given definitive therapy-surgery for 2 and radioactive iodine therapy for 5 subjects. Total of 12 subjects were lost to follow-up. The remaining subjects $(n=115)$ were continued on carbimazole and the dose was titrated to maintain euthyroidism. After completion of 18 months of ATD, it was stopped and patients were followed up with monitoring of thyroid function every 3 months to detect recurrence of thyrotoxicosis. Thyrotoxicosis recurred in 25 subjects within 3 months of stopping ATD, while recurrence happened within 6 months in another15. At the end of one year of follow up, 14 more had relapse of thyrotoxicosis.

A total of 115 patients (33 ma les, 82 females) completed the full duration of ATD therapy and one year of follow-up and thus became eligible for final analysis of results. Out of this, 61 patients were in remission for more than 12 months after stopping ATD and for med the responder group. Remission rate achieved was 53\%. The remaining 54 patients who either had persistence of thyrotoxicosis or relapse of the disease within 12 months were included in the nonresponder group (Diagram 1)

\subsection{Comparative results of responders and non-responders}

Gender - gender did not seem to have any predictive value for response to ATD.

Age - The age range of the study population was 11- 59 years with a median age of 34 years, suggesting a relatively young study group. An arbitrary value of 40 years for age was taken as the cut-off value for analysis, since subjects will generally be past the reproductive age and those above 40years may be considered as better candidates for options such as RAI. We found that $70 \%$ of the subjects were below this age at the onset of study. There was no significant difference in response to ATD in groups of subject below and above 40 years of age ( $p-$ value 0.56 ) 
Goiter size - Nearly $70 \%$ of study subjects had small goiter either grade I or II. Response to ATD was poor in those with large goiters; only $35 \%$ of those with grade III goiter went into remission with ATD while $70 \%$ of subjects with grade I and II goiters had responded $(\mathrm{p}=0.013)$.

\subsection{Free T3 levels at onset}

For sake of comparison, we used a cut-off of 20pm/L (3 times upper range of normal). While 54\% (62/115) of the study population had levels $<20 \mathrm{pm} / \mathrm{L}$ at outset, $46 \%$ had higher levels suggestive of severe thyrotoxicosis. Among the former, nearly $57 \%$ (35/61) responded compared to $50 \%$ (26/53) of those with severe thyrotoxicosis. When the groups were compared, the difference was significant $(\mathrm{p}=0.0035)$.

3.3 Free T3 levels at 3months, 6 months or 12 months

When we used this parameter $(<10 \mathrm{pm} / \mathrm{L})$ as a measure of short term response to ATD to see its relation to remission from disease, we found significant difference to exist between the two groups at $5 \%$ level of significance $(<0.0001)$. The difference between the groups continued to be significant eve $n$ when the values were compared at 6 months or 12 months.

\subsection{Free T4 levels at onset}

With $56 \mathrm{pm} / \mathrm{L}$ as the cut-off for comparison, 49 patients of the total study population at onset had values lower than this while 66 had equal or greater levels. While 29/49 in the former sub-group went into remission, 32/66 also responded, but this did not achieve statistical significance at $5 \%$ level of significance $(\mathrm{p}=0.0655)$. The differences were not significant when Mann-Whitney test was also used for comparison.

\subsection{Free T4 levels at 3months, 6 months or 12 months}

Response to ATD with FT4 levels becoming <30pm/L at 3 months was seen in 31 responders and 27 non-responders and when the groups were compared, statistical significance was obtained $(\mathrm{p}<0.0001)$. The difference in values between the two groups continued to be significant even at 6months or later at 1year. This is in contrast to insignificant difference of FT4 values at onset of study.

Results summary: Goitre size, FT3 value at first contact and at 3 and 6 months, FT4 values at 3 or 6 months all have predictive value to predict response to ATD therapy in patients with Graves' hyperthyroidism. Bigger size of goiter and higher levels of FT3 at onset and at 3 and 6 months was predictive of lower probability of remission to ATD therapy. Similarly higher levels of FT4 at onset and at 3 and 6 months had similar predictive value.

\section{Discussion}

In this study, we treated 115 patients of Graves' disease with anti-thyroid drug carbimazole for 18months and followed them up for up to twelve months without medication. Within s ix months, 40 had recurrence of thyrotoxicosis while another 14 relapsed within one year of stopping therapy. No death or major adverse effects from antithyroid medication were seen during the study. The relapse rate was 40/115 (34.8\%) within 6months and 54/115 (46. 9\%) at one year of stopping ATD.

The study population was relatively young with a median age of 34 years. The female: male ratio was 2.48:1 showing female preponderance but this was relatively less compared to 5:1 to 10:1 reported from other studies [1].

\subsection{Relapse rate vs. duration of ATD by titration regimen}

Studies have varied duration of study from 6-42months and compared short vs. long duration. Re lapse rate was $58 \%$ for 6 months ATD vs. $37 \%$ for 18 months ATD at 1year of follow up and the difference was significant as per one study by Allannic et al [11]. With longer duration of antithyroid medication, studies by Garcia-Mayor or Maugendre did not find any significant difference between 12 vs. 24 months or 18 vs. 42 months respectively with relapses ranging from 44- 50\% [12], [13].

Available evidence supports the use of titration regimen for duration of up to 12-18months as was reported in a recent Cochrane review [14] and is further corroborated in the present study. Longer duration of use does not confer any additional benefit, though many patients who have relapsed in our study have preferred to be on low dose carbimazole (ranging from 5-15mg/day) and many have been maintained biochemically euthyroid. 


\subsection{Relapse rate vs. ATD regimen used (Block-replace vs. titration regimen)}

A Cochrane review which analyzed relapse rates from different published studies found better results with blockreplace $(B R)$ regimen $(R R=50 \%)$ compared to that of $67 \%$ with titration regimen [14]. But the review did not find any significant difference when drop-outs were included in analysis assuming relapse in all the dropouts. When all studies were combined disregarding duration of follow up, re lapse rates were similar in both groups. We did not use the BR regimen due to the high dosages of carbimazole and the resultant increase in the incidence of adverse effects especially the graver ones such as agranulotcytosis. A systematic review of many trials of BR drug therapy did not find any benefit [15].

\subsection{Factors that influence response to ATD therapy}

This has been a continuing area of research ever since the introduction of antithyroid drugs several decades ago, though no $s$ ingle test or combination of tests have been identified that could predictably differentiate those patients who will respond to antithyroid drugs. This would have avoided the predicament of administering ATD for 12-18 months and then following them up to detect relapse. Out of several factors that have been studied, size of goiter, serum thyroid hormone levels or their ratios (T3/T4 ratio), thyroglobulin/TRAb levels have been extensively studied.

Presence of a large goiter or a positive TSAb test before discontinuation of ATD seem to be the best possible predictors of relapse of hyperthyroidism but even these lack adequate sensitivity or specificity [17],[16].

Relapse is more likely in men, older patients and patients with more active disease as evident from large goiter and higher concentrations of T3 and T4 as reported by Allahabadia et al [17]. In their study, gender and age at presentation were found to influence the chances of remission when treated with either carbimazole or radioiodine in a large cohort from the UK.

One study from Mexico found a high relapse rate of $82.5 \%$ after treatment with methimazole (MMZ), within a mean follow up of $11.9+/-11.8$ months [18]. They concluded that goiter size was the only statistically significant remission sign whereas other factors such as age, disease duration, MMZ dose, exophthalmos, treatment duration or concomitant use of 1-thyroxine were not useful predictive factors.

Suppression with T3 was found to be the best and cheapest predictor of outcome though this had only an accuracy of $70 \%$ for both positive and negative tests, as was reported by the Scottish Automated Follow-Up Register Group [5].

A retrospective study of patients with GD and TNG (toxic nodular goiter) by Bolanos et al evaluated the factors that determined the short-term response to treatment after 4-6 weeks with CBZ [19]. It was only 50\% in patients younger than 30 years while this was $86 \%$ in patients over the age of 30 . Other determinants of the response to carbimazole expressed as the fall in thyroid hormone levels after 4-6 weeks were: pretreatment levels of FT4, T3, TRAb and the $4 \mathrm{~h}$ 131I uptake, patients with the higher levels responding significantly better to CBZ, but this study included patients with GD treated with either CBZ or RAI and those with MNG.

Our study was a prospective one and included only those with GD. We used a standard regimen of ATD for duration of 18 months and followed them up for at least 12 months, as this is the period within which maximum of patients relapse. Analysis of our findings showed that goiter size, free hormone values at onset (FT3) and both FT3 and FT4 at 3 months and 6months to have a significant bearing on re lapse after a course of ATD. This is important in a country like India, where simple clinical parameters have to be relied upon to decide the option of therapy. Advanced diagnostic tests other than FT3, FT4 and TSH are not widely available. It may also be noted that grading of Graves' ophthalmopathy by NOSPECS was not analyzed as this will be in clinical practice only in endocrine referral centres such as ours.

In addition, inability to control thyrotoxicosis with sufficient dosage of ATD within a maximum of 6 months is an indication to consider alternative options of either surgery or RAI. Prolonged course of ATD has been shown to have a negative impact on the chances of remission with RAI[20].

The choice of therapy for GD in clinical practice is often determined by extraneous factors such as preference or experience of the clinician, local availability of centers with experience with surgery/RAI, cultural factors, cost and most importantly the awareness of not only the options but also the current guide lines recommended by professional societies. The existing gap in translation of available evidence based recommendations into clinical practice is apparent in our own centre. Though the Royal College of Physicians of United Kingdom recommended a minimum dose of 10 millicuries ( $\mathrm{mCi}$ ) of RAI for GD in 1995, experience from our center on RAI showed that a significant proportion of patients with GD were prescribed doses of RAI below $5 \mathrm{mCi}[21]$. 


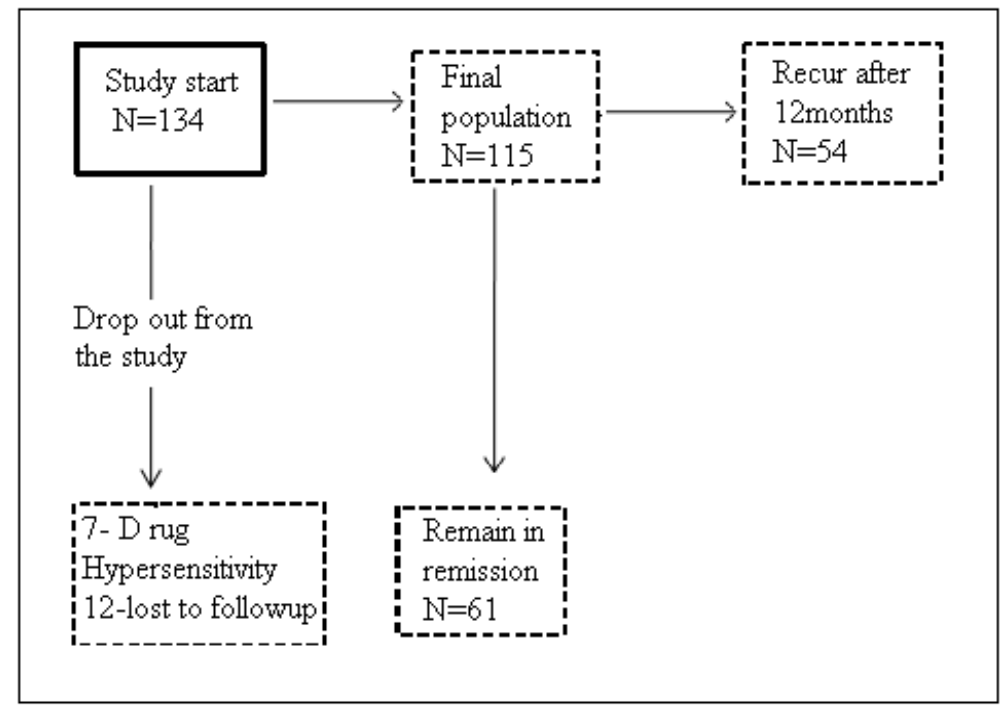

Fig.1: Schema of the study protocol and follow up of patients with graves' hyperthyroidism treated with carbimazole

\section{References}

[1] A.P. Weetman, Graves' disease, N Engl J Med 343 (2000) 1236-1248.

[2] L. Wartofsky, D. Glinoer, S.Solomon, R. Nagataki, R. Lagasse, Differences and similarities in the diagnosis and treatment of Graves' disease in Europe, Japan and the United States, Thyroid (1991)129-135.

[3] D. Glinoer, D. Hesch, R. Lagasse, P. Laurberg, The management of hyperthyroidism due to Graves'disease in Europe in 1986. Results of an international survey, Acta Endocrinol S 285(1987) 4-23.

[4] L.Wartofsky, Low remission after therapy for Graves' disease: possible relation of dietary iodine with antithyroid therapy results, J Am Med Assoc 226(1973)1083-1088

[5] A.J .Hedley, R.E. Young, S.J. Jones, W.D. Alexander, P.D. Bewsher, Scottish automated follow -up register group, Antithyroid drugs in the treatment of hyperthyroidism of Graves' disease: long term follow-up of 434 patients, Clin Endocrinol (Oxf) 31(1989) 209-218.

[6] H. Schleusner, J. Schwander, C.Fischer et al, Prospective multicenter study on the prediction of relapse after antithyroid dr ug treatment in patients with Graves' disease, Acta Endocrinol (Copenh) 120(1989) 689-701.

[7] P. Vitti, T. Rago, L. Chiovato, Clinical features of patients with Graves' disease undergoing remission after antithyroid drug treatment, Thyroid 7(1997) 369-375.

[8] World Health Organisation. Indicators for assessing iodine deficiency disorders and their control programs through salt iodination. WHO/UNICEF/ICCIDD Report 1994.

[9] S.C. Werner, Modification of the classification of the eye changes in Graves' disease: recommendations of the ad hoc committee of the American Thyroid Association, J Clin Endocrinol Metab 44 (1977)203-209.

[10] D.A. Meier, M.M. Kaplan. Radioiodine uptake and thyroid scintiscanning. Endocrinol Metab Clin N Am 30(2001) 291-313.

[11] H. Allannic, R. Fauchet, J. Orgiazzi et al, Antithyroid drugs and Graves' disease: a prospective randomized evaluation of the efficacy of treatment duration, J Clin Endocrinol Metab 70(1990) 675-679.

[12] R.V. Garcia Mayor, C. Paramo, R. LunaCano, L.F. Perez Mendez, J.C. Galofre, A. Andrade, Antithyroid drug and Graves' hyperthyroidism .Signif icance of treatment duration and TRAb determination on lasting remiss ion, J Endocrinol Inv 15(1992)815-820.

[13] D. Maugendre, A. Gatel, L. Campion et al, Antithyroid drugs and Graves' disease - a prospective randomized assessment of long term treatment. Clin Endocrinol 50(1999)127-132.

[14] P. Abraham, A. Avenell, W.A. Watson, C.M. Park, J.S. Bevan. A systematic review of drug therapy for Graves' hyperthyroidism. Cochrane Database of Systematic Reviews 2 (2005) CD 003420 (DOI: 10.1002/14651858).

[15] P. Abraham, A. Avenell, C.M. Park, W.A. Watson, J.S. Bevan. A systematic review of drug therapy for Graves' hyperthyroidism. Eur J Endocrinol 153(2005) 489-498.

[16] J.N. Talbot, F. Duron, R. Feron, P. Aubert, G. Milhaud, Thyroglobulin, thyr otropin and thyrotropin binding inhibiting immunoglobulins assayed at the withdrawal of antithyr oid drug therapay as predictors of relapse of Graves' disease within one year. J Endocrinol Inv 12 (1989)589-595.

[17] A. Allahabadia, J. Daykin, R.L. Holder, Mc. Sheppard, S.C.L. Gough, J.A. Franklyn. Age and gender predict the outcome of treatment for Graves' hyperthyroidism. J Clin Endocrinol Metab 85 (2000)1038-1042.

[18] F. Bolaños, M. Gonza lez-Ortiz, H. Duron, C. Sanchez. Remission of Graves' hyperthyroidism treated with methimazole.Rev Inv Clin 54(2002) 307-310

[19] I.M. Bringmann, B.L. van Leeuwen, G. Hennemann, G.J. Beckett, A.D. Toft, Outcome of treatment of hyperthyroidism, J Endocrinol Inv 22(1999) 250-256.

[20] C. Marcocci, D. Gianchecchi, I. Masini et al, A reappraisal of the role of methimazole and other factors on the efficacy and outcome of radioiodine therapy of Graves' hyperthyroidism. J Endocrinol Inv 13(1990)513-520.

[21] R. Sankar, T. Sekhri, G. Sripathy, R.P. Walia, S.K. Jain, Radioiodine therapy in Graves' hyperthyroidism: a prospective study from a tertiary referral centre in North India. J Assoc Physic Ind 53(2005) 603-606. 